\title{
Micromagnetic Approach to Exchange Bias
}

\author{
J. DubowiK ${ }^{a, *}$ AND I. GościańsKA ${ }^{b}$ \\ ${ }^{a}$ Institute of Molecular Physics, Polish Academy of Sciences, M. Smoluchowskiego 17, PL-60-179, Poznań, Poland \\ ${ }^{b}$ Faculty of Physics, A. Mickiewicz University, Umultowska 85, PL-61-614, Poznań, Poland
}

\begin{abstract}
We present a micromagnetic approach to the exchange bias in ferromagnetic/antiferromagnetic thin film systems with a small number of irreversible interfacial magnetic moments. We express the exchange bias field $H_{\mathrm{EB}}$ in terms of the fundamental micromagnetic length scale of ferromagnetic - the exchange length $l_{\text {ex }}$. The benefit from this approach is a better separation of the factor related to the ferromagnetic layer from the factor related to the ferromagnetic/antiferromagnetic coupling at interfaces. Using this approach we estimate the upper limit of $H_{\mathrm{EB}}$ in real ferromagnetic/antiferromagnetic systems.
\end{abstract}

DOI: 10.12693/APhysPolA.127.147

PACS: $75.30 . \mathrm{Gw}, 75.30 . \mathrm{Et}, 75.60 . \mathrm{Jk}$

\section{Introduction}

The coupling between a ferromagnet (FM) and an antiferromagnet (AFM) that is set up on field cooling from temperatures above the Néel temperature of the AFM results in an exchange bias (EB) [1]. However, it seems that we do not yet have a general and compact micromagnetic description of EB in spite of a number of numerical simulations [2-4] and models $[5,6]$. In this respect, three main points need to be emphasized.

(i) In numerous proposed mesoscopic and microscopic models of EB [5, 6], the master formula for the unidirectional anisotropy field $H_{\mathrm{EB}}$ (the exchange bias field) is

$$
H_{\mathrm{EB}}=\frac{J_{\mathrm{EB}}}{M t_{\mathrm{FM}}},
$$

where $J_{\mathrm{EB}}$ is the interfacial exchange bias energy and $t_{\mathrm{FM}}$ is the thickness of the FM layer with magnetization $M$. Equation (1) represents a relation expressing the equilibrium between the exchange bias energy density $J_{\mathrm{EB}} / t_{\mathrm{FM}}$ and the Zeeman energy [1]. The main problem is $J_{\mathrm{EB}}$, which according to us is determined by the fundamental parameters of a ferromagnet, taking into account the peculiarities of the interface structure, and of the antiferromagnet. Here, we will focus on the role of a ferromagnetic layer. A ferromagnet with a high magnetization and a high exchange stiffness gives usually the highest $H_{\mathrm{EB}}$.

(ii) An important step forward in explaining the magnitude of the EB has been done by Stöehr group, who, using X-ray magnetic circular dichroism, showed that $\mathrm{EB}$ is produced by a small $(\approx 0.04=4 \%)$ number of irreversible AFM spins $[7,8]$. Therefore, a spin structure at an FM/AFM interface consists of two groups. First, the uncompensated AFM spins - weakly coupled to the rest of the AFM spin lattice so that they can rotate. Second, the irreversible spins - a small fraction of uncompensated spins that are tightly coupled to the AFM spin lattice. Hence, a reduction factor $\epsilon$ equal

\footnotetext{
* corresponding author; e-mail: dubowik@ifmpan.poznan.pl
}

to the fraction of irreversible spins should be taken into account if Eq. (1) is to explain the experimental data.

(iii) In FM/AFM bilayers, the coercive field $H_{\mathrm{C}}$ of the FM undergoes a substantial increase by a factor of 10-20 in comparison with a single FM film due to an anisotropy $\langle K\rangle$ imposed on the FM by the AFM's uncompensated spins [5]. However, after inspecting a large number of available experimental data $[9,10]$, it appears that the saturation field $H_{\mathrm{S}}$ (measured in the hard direction) of the FM coupled to an AFM is a more reliable quantity than $H_{\mathrm{C}}$ measured in the easy direction.

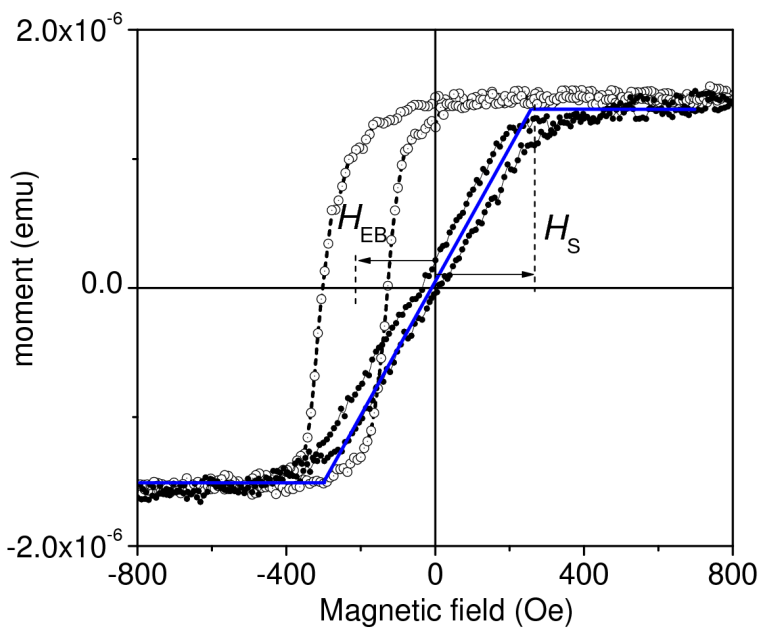

Fig. 1. Magnetic moment versus magnetic field $(H)$ curves of a sample with the following layer structure: Si (substrate) $/ \operatorname{IrMn}(20 \mathrm{~nm}) / \operatorname{Co}(4 \mathrm{~nm}) / \operatorname{IrMn}(20 \mathrm{~nm})$. Dashed line and open circles: field parallel to the exchange bias field. Full circles and thin (blue on-line) line: field perpendicular to the exchange bias field. The film was prepared under the same conditions as described in Ref. [11].

Figure 1 serves as a typical example showing that $H_{\mathrm{S}}$ is of the order of $H_{\mathrm{EB}}$. Therefore, the uniaxial anisotropy field $H_{\mathrm{S}}$ is

$$
H_{\mathrm{S}}=\frac{2\langle K\rangle}{M} \text {. }
$$


The aim of the present paper is to express Eq. (1) in a more fundamental form involving the micromagnetic characteristics of the FM. It should be stressed that by using magnetic measurements of FM/AFM systems with $\mathrm{EB}$, we can only determine the magnetic properties of the FM. Nevertheless, looking from the FM side, we can argue about an interaction between the FM and the AFM. We shall concentrate on the most important aspects of $\mathrm{EB}$, namely the main factors that determine the values of $H_{\mathrm{EB}}$ and $J_{\mathrm{EB}}$. In particular, we shall determine the role of the FM layer and we shall look for an $\mathrm{FM} / \mathrm{AFM}$ system that fulfils the requirements of an almost ideal EB effect.

\section{Model}

Three quantities describe the micromagnetism of ferromagnets: the magnetization $M$, the magnetic anisotropy $K$, and the exchange stiffness constant $A$. Since the exchange interactions are relevant for a long range spin ordering, we postulate that the last quantity is mainly responsible for interactions between the FM and AFM layers. In the first approximation, let us assume that an $\mathrm{FM} / \mathrm{AFM}$ interface is ideal, so that the interfacial spins are fully coupled. It can be easily derived from the definition of the exchange interaction in the Heisenberg approach that $J_{\mathrm{EB}}$ can be approximated by $2\langle A\rangle / \xi$, where $\langle A\rangle$ is the average exchange stiffness of an FM layer within an interface region with a thickness $\xi$ of the order of the lattice parameter [2]. Hence Eq. (1) takes the form

$$
H_{\mathrm{EB}}=\frac{2\langle A\rangle}{M \xi t_{\mathrm{FM}}} .
$$

Equivalently, the micromagnetic characteristics of an FM can be expressed in terms of the exchange length $l_{\text {ex }}$ and the exchange correlation length $l_{\text {cor }}$ (domain wall parameter) defined as

$$
\begin{aligned}
& l_{\mathrm{ex}}=\sqrt{\left(A / 2 \pi M^{2}\right)}, \\
& l_{\mathrm{cor}}=\sqrt{(A / K)},
\end{aligned}
$$

respectively. Both $l_{\mathrm{ex}}$ and $l_{\text {cor }}$ are the fundamental length scales that control the behavior of magnetic materials and are relevant for the description of an inhomogeneous orientation of the spin structure [12]. In Table we gathered the values of the magnetic polarization $4 \pi M$ and the exchange stiffness $A$ necessary for the estimation of $l_{\text {ex }}$ for the typical soft magnetic materials, some Heusler alloys, and magnetite. The values of $l_{\mathrm{ex}}$ are within the range of $3-8 \times 10^{-7} \mathrm{~cm}=3-8 \mathrm{~nm}$, while $l_{\text {cor }}$ (of the order of the Bloch wall thickness) varies considerably from $l_{\text {cor }} \approx 1 \mathrm{~nm}$ in hard magnetic materials to over $100 \mathrm{~nm}$ in soft ferromagnets [12]. The spin-wave stiffness $D=2 g \mu_{\mathrm{B}} A / M$ is also included for comparison, since both $A$ and $D$ are frequently used to describe the stiffness of exchange interactions. $g$ is the Landé $g$-factor and $\mu_{\mathrm{B}}$ is the Bohr magneton. The $g$ values of pure metals $g=2.09(\mathrm{Fe}), g=2.18(\mathrm{Co})$, and $g=2.21(\mathrm{Ni})$ deviate from the spin-only value by some amount $[7,13]$. The same concerns the Heusler alloys $(g=2.05-2.0)[14,15]$ and magnetite $(g=2.12)$.

By multiplying and dividing Eq. (3) by $4 \pi M$, we can express it in a different way

$$
H_{\mathrm{EB}}=4 \pi M \frac{\langle A\rangle}{2 \pi M^{2}} \frac{1}{\xi t_{\mathrm{FM}}}=4 \pi M \frac{\left\langle l_{\mathrm{ex}}\right\rangle^{2}}{\xi t_{\mathrm{FM}}},
$$

where $\left\langle l_{\text {ex }}\right\rangle$ denotes an averaged exchange length within the interface region. For a typical value of $l_{\mathrm{ex}}=5 \mathrm{~nm}$ (see Table), Eq. (5) leads to an unrealistically high value of $H_{\mathrm{EB}} \approx 170 \mathrm{kOe}$ if we assume typical values for $4 \pi M \approx$ $10 \mathrm{kG}, \xi \approx 3 \times 10^{-8} \mathrm{~cm}$, and $t_{\mathrm{FM}}=l_{\mathrm{ex}}$.

TABLE

Basic magnetic parameters of ferromagnetic $3 d$ metals, typical soft magnetic alloys, some Heusler alloys and magnetite $\left(\mathrm{Fe}_{3} \mathrm{O}_{4}\right)$ : the demagnetizing field $4 \pi M$, the exchange stiffness constant $A$, the spin-wave stiffness $D$, the exchange length $l_{\text {ex }}$, and the product of demagnetizing field and the square of exchange length.

\begin{tabular}{c|c|c|c|c|c}
\hline \hline Material & $\begin{array}{c}4 \pi M \\
{[\mathrm{kOe}]}\end{array}$ & $\begin{array}{c}A \\
{[\mu \mathrm{erg} / \mathrm{cm}]}\end{array}$ & $\begin{array}{c}D \\
{\left[\mathrm{meV} \mathrm{nm}^{2}\right]}\end{array}$ & $\begin{array}{c}l_{\text {ex }} \\
{[\mathrm{nm}]}\end{array}$ & $\begin{array}{c}4 \pi M l_{\text {ex }}^{2} \\
{\left[\mathrm{GOe} \mathrm{cm}^{2}\right]}\end{array}$ \\
\hline $\mathrm{Fe}$ & $21.4^{a}$ & $2.0^{b}$ & 2.8 & 3.3 & 2.3 \\
$\mathrm{Co}$ & $18.1^{a}$ & $2.5^{b}$ & 4.5 & 4.4 & 3.5 \\
$\mathrm{Ni}$ & $6.1^{a}$ & $0.8^{b}$ & 4.5 & 7.6 & 3.5 \\
$\mathrm{Ni}_{80} \mathrm{Fe}_{20}$ & $10.0^{a}$ & $1.0^{b}$ & 2.5 & 5.0 & 2.5 \\
$\mathrm{Co}_{47} \mathrm{Fe}_{53}$ & $17.0^{c}$ & $5.9^{c}$ & 8.0 & 7.2 & 8.7 \\
$\mathrm{Co}_{2} \mathrm{FeSi}$ & $14.1^{d}$ & $3.2^{d}$ & 7.0 & 6.4 & 5.7 \\
$\mathrm{Co}_{2} \mathrm{MnSn}$ & $9.9^{e}$ & $0.6^{e}$ & 2.0 & 3.9 & 1.5 \\
$\mathrm{Ni}_{2} \mathrm{MnSn}$ & $5.1^{f}$ & $0.1^{f}$ & 0.4 & 2.6 & 0.3 \\
$\mathrm{Fe}_{3} \mathrm{O}_{4} h$ & $5.9^{g}$ & $0.7^{g}$ & 5.0 & 7.1 & 3.0 \\
\end{tabular}

${ }^{a}$ from Ref. [16], ${ }^{b}$ from Ref. [13], ${ }^{c}$ from Ref. [17], ${ }^{d}$ from Ref. [14], ${ }^{e}$ from Ref. [18], ${ }^{f}$ from Ref. [15], ${ }^{g}$ from Ref. [19], ${ }^{h}$ all data are representative for room temperature except that of magnetite, which is at $5 \mathrm{~K}$.

The comment (ii) implies that $\langle A\rangle$ and $\left\langle l_{\text {ex }}\right\rangle$ are to some extent weakened by the low number of the pinned spins. Let us inspect the impact of the low number of the irreversible spins on $\langle A\rangle$ more closely. If we imagine the interface shown in Fig. 2 with the spins (marked by circles) pinned to the rest of the AFM (marked by shaded area), we can see that they are exchange-coupled with equal numbers $\epsilon$ of FM spins. Therefore,

$$
\langle A\rangle \approx \frac{I \epsilon S_{\mathrm{FM}} \epsilon S_{\mathrm{AFM}}}{a} \approx \epsilon^{2} A,
$$

where $I$ is the exchange integral. $S_{\mathrm{FM}}$ and $S_{\mathrm{AFM}}$ are the FM and AFM spins, respectively. Here we assume that the EB systems exhibit negative bias, so that the FM spins and irreversible AFM spins are aligned in the same direction $(I>0)$ [8]. Hence, an EB for a realistic interface with a low number of irreversible spins can be expressed by

$$
H_{\mathrm{EB}}=4 \pi M l_{\mathrm{ex}}^{2} \frac{\epsilon^{2}}{\xi t_{\mathrm{FM}}}
$$

with the product of $4 \pi M l_{\mathrm{ex}}^{2}$ as the leading factor. From Table one can see that the leading factor is highest for the $\mathrm{Co}-\mathrm{Fe}$ alloy and, unexpectedly, for the $\mathrm{Co}_{2} \mathrm{FeSi}$ Heusler 
alloy, while it does not vary much for $3 d$ metals and $\mathrm{Ni}-\mathrm{Fe}$. It is easy to show that an $\epsilon$ on the order of a few percent provides a realistic value of $H_{\mathrm{EB}} \approx 200 \mathrm{Oe}$ $(\epsilon=0.015)$, as is shown in Fig. 1, for example, and in agreement with other experimental data (see, for example Ref. [5] and references therein). The expression $\left\langle l_{\mathrm{ex}}\right\rangle=\epsilon l_{\mathrm{ex}}$ can be regarded as an effective exchange length in FM due to the low number of irreversible AFM spins. Note, however, that $\left\langle l_{\mathrm{ex}}\right\rangle$ is on the order of only $0.1 \mathrm{~nm}$ if $\epsilon=0.04$. This is a remarkably small value since, by definition, the exchange length is the length below which atomic exchange interactions dominate over typical magnetostatic fields [12].

Accordingly, the interfacial exchange bias energy $J_{\mathrm{EB}}$ is expressed by

$$
J_{\mathrm{EB}}=4 \pi M^{2}\left(\frac{l_{\mathrm{ex}}^{2} \epsilon^{2}}{\xi}\right),
$$

where the second factor in parentheses has the dimension of length scale of $200 \mathrm{~nm}$ if $\epsilon=1$ so that $J_{\mathrm{EB}}$ would take a huge value of $50-150 \mathrm{erg} / \mathrm{cm}^{2}$. However, since $J_{\mathrm{EB}}$ must be less than $K_{\mathrm{AFM}} \times t_{\mathrm{AFM}}$ [1], the upper limit of $J_{\mathrm{EB}}$ is less than $10 \mathrm{erg} / \mathrm{cm}^{2}$ if $K_{\mathrm{AFM}} \approx 10^{7} \mathrm{erg} / \mathrm{cm}^{3}$ and $t_{\mathrm{AFM}} \approx$ $5 \mathrm{~nm}$ (a typical critical value of AFM thickness) [20]. It is noteworthy that if $\epsilon=0.35$ and $0.04, J_{\mathrm{EB}}$ would be $10-4$ and $0.4-0.1 \mathrm{erg} / \mathrm{cm}^{2}$, respectively.

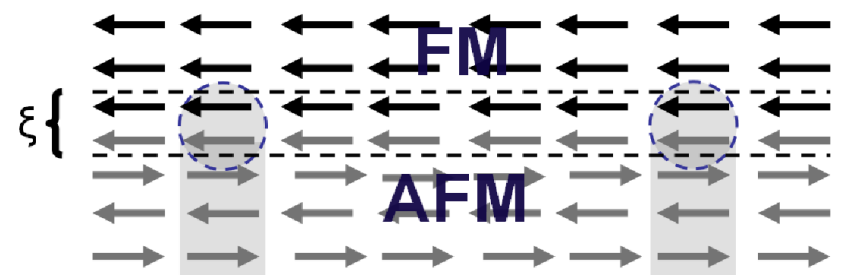

Fig. 2. Schematic diagram of the FM/AFM interface of thickness $\xi$ with uncompensated spins and the spins (marked by dashed circles) pinned to the AFM spin lattice (shaded area).

\section{Discussion}

The lowest limit of $J_{\mathrm{EB}}$ of $0.1-0.4 \mathrm{erg} / \mathrm{cm}^{2}$ is worth comment. Since research into EB encompasses a huge number of papers, we can draw useful conclusions on the average exchange bias energy $J_{\mathrm{EB}}$ using simple statistics for a large number of experimental data under the assumption that each experiment is of equal significance. A collection of tabulated data gathered by Coehoorn [20] is an invaluable database. We gathered the distribution of the values of $J_{\mathrm{EB}}$ (taken from Tab. 13 in Ref. [20]) in the form of histograms as shown in Fig. 3. It is clearly seen that both for $\mathrm{Ni}-\mathrm{Fe}$ and $\mathrm{Co}-\mathrm{Fe}$ layers in contact with various metallic random substitutional fcc-type AFM alloys, the distributions of the data have the shape of a normal distribution even though the width of the histogram for the $\mathrm{Co}-\mathrm{Fe}$ data is four times higher than that of the Ni-Fe data. Most important, however, is that the mean value of $J_{\mathrm{EB}}$ is 0.1 and $0.25 \mathrm{erg} / \mathrm{cm}^{2}$ for $\mathrm{Ni}-\mathrm{Fe}$ and
$\mathrm{Co}-\mathrm{Fe}$, respectively. If we estimate the values of $J_{\mathrm{EB}}$ making use of Eq. (8) assuming $\epsilon=0.04$, we unexpectedly arrive at $J_{\mathrm{EB}}=0.11$ and $0.24 \mathrm{erg} / \mathrm{cm}^{2}$, nearly the same as the mean values evaluated from the histograms for $\mathrm{Ni}-\mathrm{Fe}$ and $\mathrm{Co}-\mathrm{Fe}$, respectively. For the calculations, we took the appropriate data from Table and $\xi=0.3$ and $0.25 \mathrm{~nm}$ for permalloy and Co, respectively. The remarkable agreement between the experimental and calculated values of $J_{\mathrm{EB}}$ may seem a coincidence, but it may also suggest that the assumed ratio of the irreversible pinned spins of just a few percent is typical of metallic FM/AFM systems. One of the highest values of $J_{\mathrm{EB}}$ ever measured for metallic FM/AFM bilayers (with a high $4 \pi M l_{\mathrm{ex}}^{2}$ factor) is $1.3 \mathrm{erg} / \mathrm{cm}^{2}$ for $\mathrm{Co}_{70} \mathrm{Fe}_{30}$ in contact with a highly $\mathrm{L1}_{2}$ ordered $\mathrm{Mn}_{3}$ Ir phase [21]. A rough estimate employing Eq. (8) suggests that such a "giant" value is achieved for a merely twofold increase in $\epsilon$ to $0.08-0.09$.

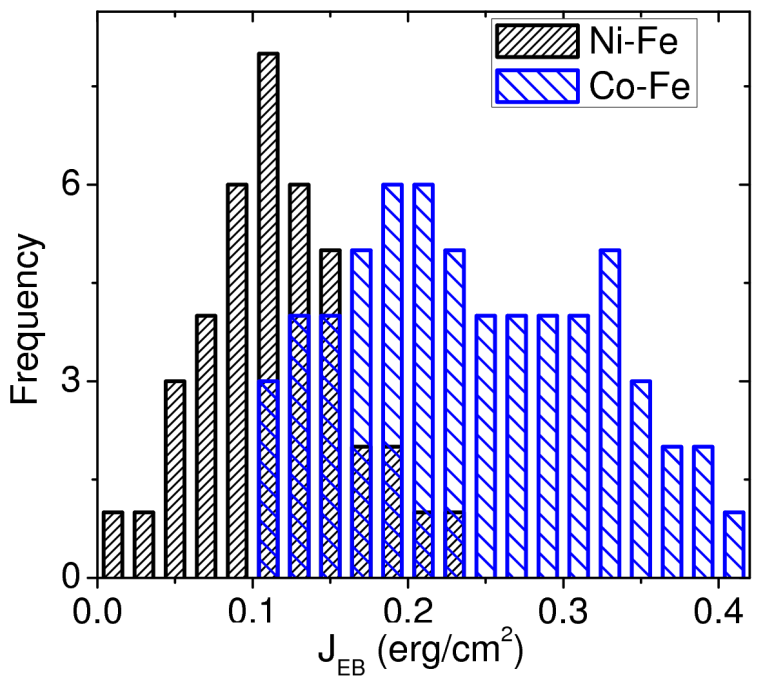

Fig. 3. Histograms showing distribution of the experimental values of $J_{\mathrm{EB}}$ taken from Tab. 13 in Ref. [20]. The histograms show distribution of the data for Ni-Fe $\left(\mathrm{Ni}_{80} \mathrm{Fe}_{20}\right)$ and for $\mathrm{Co}-\mathrm{Fe}$ films $\left(\mathrm{Co}_{90} \mathrm{Fe}_{10}\right)$, respectively. Frequency has the meaning of the number of data falling in a bin of $J_{\mathrm{EB}}$.

Therefore, the FM/AFM systems behave as if a specific localized exchange coupling between $\epsilon$ fraction of FM spins and an equal fraction of AFM irreversible spins were blurred in a delocalized "sea" of FM interacting spins. In this aspect, EB can be regarded as a perturbation in the exchange energy of the FM in contact with AFM.

Still, Eq. (7) describes an idealized case of the exchange bias. In reality, in most cases both the FM and the AFM have a large number of defects: they consist of grains on a nanometer scale with grain boundaries, etc. Nevertheless, Eq. (7) captures the most important material and interface characteristics that determine the order of magnitude of the exchange bias on the nanoscale. The most characteristic in Eq. (7) is that the factor $4 \pi M l_{\mathrm{ex}}^{2}$ depends exclusively on the FM (due to our assumption that the interface coupling between the irreversible AFM spins and the FM spins is positive), while the factor $\epsilon^{2}$ 
depends mostly on the AFM (its anisotropy) and the quality of the interface.

By applying the same transformation to Eq. (2) as to Eq. (5), we have

$$
H_{\mathrm{S}}=4 \pi M \frac{\langle K\rangle}{2 \pi M^{2}} \frac{\epsilon^{2} A}{\epsilon^{2} A}=4 \pi M l_{\mathrm{ex}}^{2} \frac{1}{l_{\mathrm{cor}}^{2}},
$$

which has the same symmetrical form as Eq. (7) with $l_{\text {cor }}^{2}$ in the denominator. It is characteristic that the factor $\epsilon^{2}$ is absent. For a typical value of $H_{\mathrm{S}}=200$ Oe in Fig. 1, Eq. (9) leads to $l_{\text {cor }}=32 \mathrm{~nm}$.

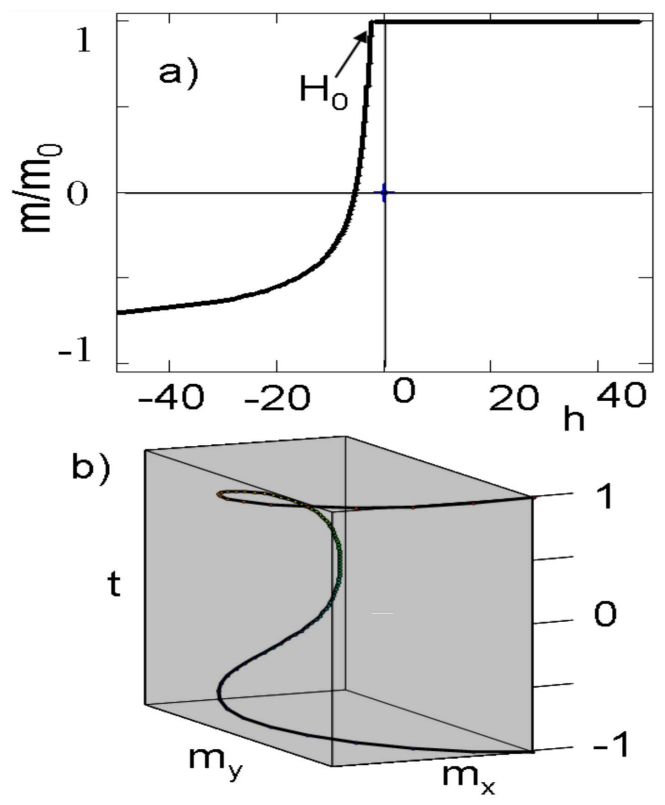

Fig. 4. (a) Asymmetrical dependence of reduced magnetization $m$ versus reduced magnetic field $h$ applied opposite to the pinning direction. (b) Twisting of the magnetization vector defined as $\boldsymbol{m}=\boldsymbol{i} \cos \Theta(t)+\boldsymbol{j} \sin \Theta(t)$ with the distance between two FM/AFM interfaces for $h=0.5$. The magnetization is fully pinned at $t= \pm 1$.

The presence of both $l_{\text {ex }}$ and $l_{\text {cor }}$ in Eqs. (7) and (9) may be linked with an inhomogeneous spin structure of the FM and suggests the formation of a magnetization twisting. Such a magnetization twisting was analyzed about 50 years ago by Aharoni et al. [22]. They considered a ferromagnetic slab, infinite in the $x$ and $y$ directions and of width $2 t_{\mathrm{FM}}$. At $t=z / t_{\mathrm{FM}}= \pm 1$, the spins are assumed to be held in the $x$ direction by the exchange coupling with the AFM and expressed with appropriate boundary conditions. Let an external field $H$ be applied in the $x$ direction. The easiest mode for magnetization changes is evidently rotation of the spins in the $x y$ plane. The functions which minimize the energy of such a system are the solutions of the Euler equation

$$
\frac{\mathrm{d}^{2} \Theta}{\mathrm{d} t^{2}}-\frac{h}{\left(t_{\mathrm{FM}} / l_{\mathrm{ex}}\right)^{2}} \sin \Theta=0,
$$

with the boundary conditions $\Theta^{\prime}(0)=0, \Theta( \pm 1)=0$ and with $h=H / 4 \pi M$. The solution of Eq. (10) is expressed in terms of the complete elliptic integral of the first kind $K_{\mathrm{C}}$

$$
\Theta(t)=2 \arcsin \left(k \operatorname{sn}\left((1-t) K_{\mathrm{C}}(k), k\right)\right),
$$

where sn is the sine amplitude function and $k$ is "hidden" in the relation $-h=K_{\mathrm{C}}^{2}(k) /\left(t_{\mathrm{FM}} / l_{\mathrm{ex}}\right)^{2}$. This solution leads to a strongly asymmetric magnetization reversal curve as shown in Fig. $4 \mathrm{a}$, which saturates at $-h \approx \infty$. This important approach to EB has not attracted much attention except in some old papers [23]. Similar asymmetric magnetization reversals have been recently observed in $\mathrm{Ni} / \mathrm{FeF}_{2}$ bilayers and interpreted as originating from the intrinsic broken symmetry of the system, which results in local incomplete domain walls parallel to the interface (i.e., the magnetization twisting) in reversal to negative saturation of the FM [3]. The twisting of the magnetization vector shown in Fig. $4 \mathrm{~b}$ comes from the boundary conditions stating that the magnetization is fully "free" at the center of the FM layer and fully "pinned" at the two FM/AFM interfaces.

As is seen in Fig. 4a, the magnetization starts twisting above a certain field $h>(\pi / 2)^{2} /\left(t_{\mathrm{FM}} / l_{\mathrm{ex}}\right)^{2}$. Hence,

$$
H_{0}=4 \pi M l_{\mathrm{ex}}^{2} \frac{(\pi / 2)^{2}}{t_{\mathrm{FM}}^{2}} .
$$

Equation (12) describes the magnitude of the exchange bias field for an ideal FM/AFM system without uniaxial anisotropy imposed by AFM but with fully irreversible spins at the interfaces. The similarity between Eq. (12) and Eq. (7) is striking in that the factor $(\pi / 2)^{2} / t_{\mathrm{FM}}^{2}$ is purely geometrical. If we equate $H_{\mathrm{EB}}$ with $H_{0}$ (Eq. (7) to Eq. (12)) in order to estimate the maximal value that $\epsilon$ can achieve for the ideal pinning described by the boundary conditions, we obtain

$$
\epsilon_{\max }^{2}=(\pi / 2)^{2} \frac{\xi}{t_{\mathrm{FM}}} .
$$

For typical values $\xi \approx 0.3 \mathrm{~nm}$ and $t_{\mathrm{FM}} \approx l_{\mathrm{ex}} \approx 5-10 \mathrm{~nm}$, $\epsilon^{2} \approx 0.15-0.075$. As a result $38 \%-27 \%$ of the irreversible AFM spins would produce the highest possible values that $H_{\mathrm{EB}}\left(J_{\mathrm{EB}}\right)$ can achieve, i.e., $25-12 \mathrm{kOe}$ (6-3 erg $\left./ \mathrm{cm}^{2}\right)$. Hence, we come to the conclusion that the highest value that $\epsilon$ can attain is $(38 \%-27 \%)$ and is just due to the formation of an incomplete domain wall (i.e., magnetization twisting). In reality, however, AFM is polycrystalline and defected, so that these values are overestimated [4].

It appears that among a large number of $\mathrm{FM} / \mathrm{AFM}$ systems all-oxide $\mathrm{Fe}_{3} \mathrm{O}_{4} / \mathrm{CoO}$ epitaxial bilayers nearly satisfy the requirements of ideal pinning with $J_{\mathrm{EB}}=$ $2.1 \mathrm{erg} / \mathrm{cm}^{2}$ - the value is only about 8 times smaller than the exchange biasing estimated according to the Meiklejohn-Bean model $[19,24]$. $J_{\mathrm{EB}}$ estimated from Eq. (8) with $\epsilon^{2}=0.13$ (i.e., $\approx 1 / 8$ ) and $\xi \approx 0.8 \mathrm{~nm}$ (lattice parameter of $\mathrm{Fe}_{3} \mathrm{O}_{4}$ ) yields $J_{\mathrm{EB}}=2.04 \mathrm{erg} / \mathrm{cm}^{2}$, which justifies the [100] oriented $\mathrm{Fe}_{3} \mathrm{O}_{4} / \mathrm{CoO}$ bilayer system's being very close to the straightforward idea concerning the micromagnetic approach. It is noteworthy that polarized neutron reflectivity measurements of similar $\mathrm{Fe}_{3} \mathrm{O}_{4} / \mathrm{NiO}$ multilayers have provided evidence of domain-wall formation (magnetization twisting) in the exchange-biased state but within the ferromagnetic, 
rather than the AFM, layer [25]. A question may be posed: why is the EB the highest in all-oxide FM/AFM systems? Neglecting the complex interplay between the microstructure of the AFM layer and the FM/AFM interface, it seems that superexchange coupling via the intervening $p$-orbitals of the oxygen atoms plays a leading role. The coupling, between the magnetic ions with half-occupied orbitals $\left(\mathrm{Fe}^{2+}\right.$ and $\left.\mathrm{Co}^{2+}\right)$ through the intermediary oxygen ion, of the superexchange is indirect (the magnetic ions are of $0.4 \mathrm{~nm}$ apart) and strongly antiferromagnetic. What seems even more important is that both oxide lattices are based on an approximately close-packed lattice of oxygen ions with $\mathrm{Co}^{2+}$ and $\mathrm{Fe}^{3+}$ in tetrahedral or octahedral $\left(\mathrm{Fe}^{3+}\right.$ and $\left.\mathrm{Fe}^{2+}\right)$ interstitial [24]. This feature makes $\mathrm{Fe}_{3} \mathrm{O}_{4} / \mathrm{CoO}$ epitaxial bilayers a model system to study EB [19]. In contrast, in all-metallic FM/AFM systems, the exchange coupling between the FM and AFM species is direct, so that any change in ordering at the interfaces results in a frustration of exchange interactions [26].

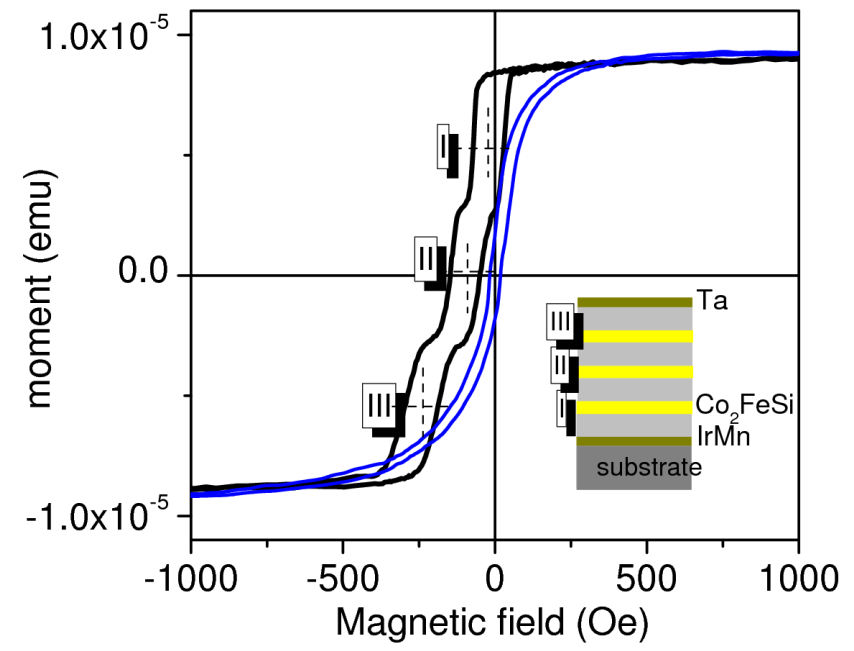

Fig. 5. Hysteresis loops of [Ta $5 \mathrm{~nm} /(\mathrm{IrMn}$ $\left.\left.20 \mathrm{~nm} / \mathrm{Co}_{2} \mathrm{FeSi} 10 \mathrm{~nm}\right) \times 3 / \operatorname{IrMn} 20 \mathrm{~nm} / \mathrm{Ta} 5 \mathrm{~nm}\right]$ multilayer taken with the magnetic field applied parallel (thick black - on-line) and perpendicular (thin blue on-line) to $H_{\mathrm{EB}}$ direction, respectively. The inset shows the multilayer structure consisting of a stack of IrMn (gray) $/ \mathrm{Co}_{2} \mathrm{FeSi}$ (yellow)/IrMn three-layers.

Now we can understand why in most of the FM/AFM all-metallic thin film systems the EB field is of the order of 100-400 Oe if $t_{\mathrm{FM}}$ is of $10 \mathrm{~nm}$ [20]. As seen in Table, the product $4 \pi M l_{\mathrm{ex}}^{2}$ does not differ much among most of the soft FM materials. Therefore, any enhancement of the EB relies mainly on increasing $\epsilon$. We have little room for manoeuvre except to increase $\epsilon$ by some technological trick like, for example, dusting the interfaces with ultrathin Co or Mn layers [11, 27], a proper setting AFM in a magnetic field [5] or preparing an AFM (e.g. IrMn) [28] with a high texture. Specifically, $\epsilon$ determines the quality of setting AFM on cooling from $T>T_{\mathrm{N}}$ [29].
A spectacular example of such a gradual improvement in EB is observed in a Ta $5 /\left(\operatorname{IrMn~} 20 / \mathrm{Co}_{2} \mathrm{FeSi} 10\right) \times 3 /$ IrMn $20 /$ Ta 5 multilayer annealed at $400^{\circ} \mathrm{C}$ for $15 \mathrm{~min}$ and field cooled to room temperature (Fig. 5). The details of the sample preparation can be found in Ref. [11]. As seen in Fig. 5, the hysteresis loop (thick line) taken with the magnetic field parallel to the exchange bias consists of three loops related to the subsequent $\mathrm{Co}_{2} \mathrm{FeSi}$ layers in the stack. These three loops, of equal heights and nearly equal coercive field of $\sim 50 \mathrm{Oe}$, are shifted along the field axis by $H_{\mathrm{EB}}$ of 20,72 and 235 Oe for the first (I), second (II) and third (III) $\mathrm{Co}_{2} \mathrm{FeSi}$ layer, respectively. Simultaneously, in accordance with the discussion of Eq. (9), the estimated value of $H_{\mathrm{S}}$ is 150 Oe (see Fig. 5, hysteresis (thin line) taken with a magnetic field applied perpendicular to $H_{\mathrm{EB}}$ direction). It has been confirmed (see Ref. [11] for details) with a magneto-optical Kerr magnetometer that the upper (III) $\mathrm{Co}_{2} \mathrm{FeSi}$ layer has the highest $H_{\mathrm{EB}}$. A simple estimate employing Eq. (7) using the data from Table for $\mathrm{Co}_{2} \mathrm{FeSi}$ gives the values of $\epsilon$ of $0.01,0.02$, and 0.05 for the layers I, II, and III, respectively. As was discussed above, $\epsilon$ depends on the interface quality and on the anisotropy of the AFM layer. Since the interfaces in the stack should not differ much, the increase in anisotropy seems to be responsible for these slight changes in $\epsilon$. We have proven with X-ray diffraction measurements (see Ref. [11]) that the grain size of IrMn increases as the subsequent layers are deposited from the substrate, so that the increase in AFM anisotropy is justified. However, in view of our discussion, we do not expect that $\epsilon$ can exceed the values of several percent in the case of all-metallic FM/AFM systems.

\section{Summary}

In summary, we have shown that the exchange bias resulting from a coupling between FM and AFM layers can be described in terms of a rough micromagnetic approach, which seems to capture the essential characteristics of the exchange bias. Specifically, we showed that the interfacial interactions involved between the FM and the AM reduce to a geometrical problem with the fundamental micromagnetic length scale being the exchange length $l_{\mathrm{ex}}$. The model identifies the range of the exchange bias field $H_{\mathrm{EB}}$ (exchange bias energy $J_{\mathrm{EB}}$ ) compatible with those observed in experiment. Using the model, we proved that the highest effective number of irreversible spins is lower than $\approx 30 \%-40 \%$.

\section{References}

[1] W.H. Meiklejohn, C.P. Bean, Phys. Rev. 102, 1413 (1956).

[2] D. Suess, M. Kirschner, T. Schrefl, J. Fidler, R.L. Stamps, J.-V. Kim, Phys. Rev. B 67, 054419 (2003).

[3] Z.-P. Li, O. Petracic, R. Morales, J. Olamit, X. Batlle, K. Liu, I.K. Schuller, Phys. Rev. Lett. 96, 217205 (2006). 
[4] M. Fecioru-Morariu, U. Nowak, G. Güntherodt, in: Magnetic Properties of Antiferromagnetic Oxide Materials, Eds. L. Dúo, M. Finazzi, F. Ciccacci, WileyVCH, Weinheim 2010, Ch. 5.

[5] J. Nogues, I.K. Schuller, J. Magn. Magn. Mater. 192, 203 (1999).

[6] A.E. Berkowitz, K. Takano, J. Magn. Magn. Mater. 200, 552 (1999).

[7] H. Ohldag, T.J. Regan, J. Stöhr, A. Scholl, F. Nolting, J. Lüning, C. Stamm, S. Anders, R.L. White, Phys. Rev. Lett. 87, 247201 (2001).

[8] J. Stöhr, H. Siegmann, Magnetism: From Fundamentals to Nanoscale Dynamics, Springer Series in SolidState Sciences, Springer, Berlin 2006, Ch. 13, p. 617.

[9] A. Kohn, J. Dean, A. Kovacs, A. Zeltser, M.J. Carey, D. Geiger, G. Hrkac, T. Schrefl, D. Allwood, J. Appl. Phys. 109, 083924 (2011).

[10] A. Harres, J. Geshev, J. Phys. Condens. Matter 24, 326004 (2012).

[11] J. Dubowik, I. Gościańska, K. Załęski, H. Głowiński, Y. Kudryavtsev, A. Ehresmann, J. Appl. Phys. 113, 193907 (2013).

[12] R. Skomski, J. Phys. Condens. Matter 15, R841 (2003).

[13] Z. Frait, D. Fraitova, in: Modern Problems in Condensed Matter Sciences, Spin Waves and Magnetic Excitations, Vol. 22.2, Eds. A.S. Borovik-Romanov, S.K. Sinha, North-Holland, Amsterdam 1988, Ch. 1, p. 1.

[14] S. Trudel, O. Gaier, J. Hamrle, B. Hillebrands, J. Phys. D Appl. Phys. 43, 193001 (2010).

[15] J. Dubowik, I. Gościańska, K. Załęski, H. Głowiński, A. Ehresmann, G. Kakazei, S.A. Bunayev, Acta Phys. Pol. A 121, 1121 (2012).

[16] R. O'Handley, Modern Magnetic Materials: Principles and Applications, Wiley, New York 1999, Ch. 3.
[17] X. Liu, R. Sooryakumar, C.J. Gutierrez, G.A. Prinz, J. Appl. Phys. 75, 7021 (1994).

[18] R. Yilgin, S. Kazan, B. Rameev, B. Aktas, K. Westerholt, J. Phys. Conf. Series 153, 012068 (2009).

[19] P.J. van der Zaag, A.R. Ball, L.F. Feiner, R.M. Wolf, P.A.A. van der Heijden, J. Appl. Phys. 79, 5103 (1996).

[20] R. Coehoorn, Lecture Notes on Novel Magnetoelectronic Materials and Devices: Exchange Biased Spinvalves, 2004, part 4 .

[21] M. Tsunoda, K. Imakita, M. Naka, M. Takahashi, J. Magn. Magn. Mater. 304, 55 (2006).

[22] A. Aharoni, E.H. Frei, S. Shtrikman, J. Appl. Phys. 30, 1956 (1959).

[23] N. Salansky, M. Eruchimov, Thin Solid Films 6, 129 (1970).

[24] P.J. van der Zaag, J.A. Brochers, in: Magnetic Properties of Antiferromagnetic Oxide Materials, Eds. L. Dúo, M. Finazzi, F. Ciccacci, Wiley-VCH, New York 2010, Ch. 7.

[25] A.R. Ball, A.J.G. Leenaers, P.J. van der Zaag, K.A. Shaw, B. Singer, D.M. Lind, H. Fredrikze, M. Rekveldt, Appl. Phys. Lett. 69, 1489 (1996).

[26] F. Radu, H. Zabel, in: Magnetic Heterostructures, series Springer Tracts in Modern Physics, Vol. 227, Eds. H.Zabel, S.D. Bader, Springer, Berlin 2008, p. 97.

[27] H. Endo, A. Hirohata, T. Nakayama, K.O. Grady, J. Phys. D Appl. Phys. 44, 145003 (2011).

[28] P. Wisniowski, T. Stobiecki, J. Kanak, G. Reiss, H. Brueckl, J. Appl. Phys. 100, 013906 (2006).

[29] K. O'Grady, L. Fernandez-Outon, G. VallejoFernandez, J. Magn. Magn. Mater. 322, 883 (2010). 